\title{
PHYLOGENETIC CONFIDENCE INTERVALS FOR THE OPTIMAL TRAIT VALUE
}

\author{
KRZYSZTOF BARTOSZEK, ${ }^{*}$ Uppsala University \\ SERIK SAGITOV, ${ }^{* *}$ Chalmers University of Technology and the University of Gothenburg
}

\begin{abstract}
We consider a stochastic evolutionary model for a phenotype developing amongst $n$ related species with unknown phylogeny. The unknown tree is modelled by a Yule process conditioned on $n$ contemporary nodes. The trait value is assumed to evolve along lineages as an Ornstein-Uhlenbeck process. As a result, the trait values of the $n$ species form a sample with dependent observations. We establish three limit theorems for the sample mean corresponding to three domains for the adaptation rate. In the case of fast adaptation, we show that for large $n$ the normalized sample mean is approximately normally distributed. Using these limit theorems, we develop novel confidence interval formulae for the optimal trait value.
\end{abstract}

Keywords: Central limit theorem; conditioned Yule process; macroevolution; martingales; Ornstein-Uhlenbeck process; phylogenetics

2010 Mathematics Subject Classification: Primary 62M05; 62P10

Secondary 60H30; 92B99

\section{Introduction}

Phylogenetic comparative methods deal with multi-species trait value data. This is an established and rapidly expanding area of research concerning evolution of phenotypes in groups of related species living under various environmental conditions. An important feature of such data is the branching structure of evolution causing dependence among the observed trait values. For this reason the usual starting point for phylogenetic comparative studies is an inferred phylogeny describing the evolutionary relationships. The likelihood can be computed by assuming a model for trait evolution along the branches of this fixed tree, such as the Ornstein-Uhlenbeck process.

The one-dimensional Ornstein-Uhlenbeck model is characterized by four parameters: the optimal value $\theta$, the adaptation rate $\alpha>0$, the ancestral value $X_{0}$, and the noise size $\sigma$. The classical Brownian motion model [14] can be viewed as a special case with $\alpha=0$ and $\theta$ being irrelevant. As with any statistical procedure, it is important to be able to compute confidence intervals for these parameters. However, confidence intervals are often not mentioned in phylogenetic comparative studies [8].

There are a number of possible numerical methods of calculating such confidence intervals when the underlying phylogenetic tree is known. Using a regression framework one can apply standard regression theory methods to compute confidence intervals for $\left(\theta, X_{0}\right)$ conditionally

Received 23 May 2014; revision received 23 October 2014

* Postal address: Department of Mathematics, Uppsala University, 75106 Uppsala, Sweden.

Email address: bartoszekkj@gmail.com

** Postal address: Department of Mathematical Sciences, Chalmers University of Technology and the University of Gothenburg, 41296 Göteborg, Sweden. 
on $\left(\alpha, \sigma^{2}\right)$ [15], [20], [28], [33]. Notably in [16] the authors derived analytical formulae for confidence intervals for $X_{0}$ under the Brownian motion model. In more complicated situations a parametric bootstrap is a (computationally very demanding) way out [8], [11], [27]. Another approach is to report a support surface [20], [21], or consider the curvature of the likelihood surface [7].

All of the above methods have in common the assumption that the phylogeny describing the evolutionary relationships is fully resolved. Possible errors in the topology can cause problems - the closer to the tips they occur, the more problematic they can be [43]. On the other hand, the regression estimators will remain unbiased even with a misspecified tree [34] and also seem to be robust with respect to errors in the phylogeny, at least for the Brownian motion model [42]. There are only a few papers addressing the issue of phylogenetic uncertainty. A Markov chain Monte Carlo procedure to jointly estimate the phylogeny and parameters of the Brownian model of trait evolution was suggested in [25] and [26]. Recently, Slater et al. [36] developed an approximate Bayesian computation framework to estimate Brownian motion parameters in the case of an incomplete tree.

In this paper we study a situation when nothing is known about the phylogeny. The simplest stochastic model addressing this case is a combination of a Yule tree and the Brownian motion on top of it: already in the 1970s, a joint maximum likelihood estimation procedure of a Yule tree and Brownian motion on top of it was proposed in [13]. This basic evolutionary model allows for far reaching analytical analysis [6], [12], [35]. A more realistic stochastic model of this kind combines the Brownian motion with a birth-death tree allowing for the extinction of species [10]. For the latter model Sagitov and Bartoszek [35] explicitly computed the so-called interspecies correlation coefficient. Such 'tree-free' models are appropriate for working with fossil data when there may be rich fossilized phenotypic information available but the molecular material might have degraded so much that it is impossible to infer evolutionary relationships. Crawford and Suchard [12] demonstrated the usefulness of the tree-free approach for contemporary species in a Carnivora order case study and Mulder and Crawford [31] calculated the distribution over the space of Yule trees of the interspecies correlation coefficient.

Conditioned birth-death processes as stochastic models for species trees have received significant attention in the last decade [3], [19], [29], [38], [39], [40]. In this paper the unknown tree is modeled by the Yule process conditioned on $n$ extant species while the evolution of a trait along a lineage is viewed as the Ornstein-Uhlenbeck process; see Figure 1. We study the properties of the sample mean and sample variance computed from the vector of $n$ trait values. Our main results are three asymptotic confidence interval formulae for the optimal trait value $\theta$. These three formulae represent three asymptotic regimes for different values of the adaptation rate $\alpha$.

Crawford and Suchard [12] pointed out that 'as evolutionary biologists further refine our knowledge of the tree of life, the number of clades whose phylogeny is truly unknown may diminish, along with interest in tree-free estimation methods.' In our opinion the main contribution of such methods is that they indicate statistical and asymptotic properties of phylogenetic samples under given evolutionary models. These properties can then be verified for other models of tree growth or real phylogenies [4], [5], [17], [22], [23], [24], [30]. Furthermore, we believe that the easy-to-compute tree-free predictions will always play the important role of a sanity check to see whether the conclusions based on the inferred phylogeny deviate much from those from a 'typical' phylogeny. Moreover, results like those presented here can also be used as a method of testing software for phylogenetic comparative models. 


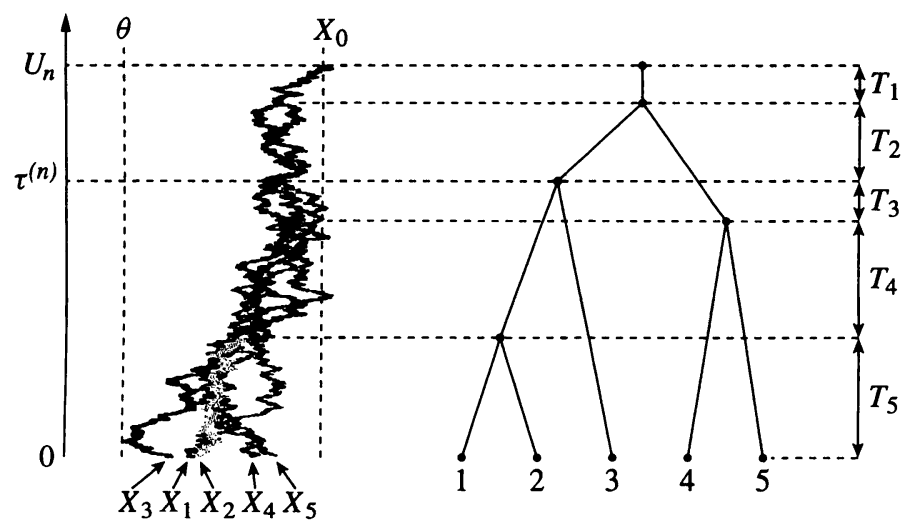

FiguRE 1: Left: A branching Ornstein-Uhlenbeck process simulated on a realization of the Yule $n$-tree with $n=5$ tips using the TreeSim [38], [39] and mvSLOUCH [7] for packages R [32]. Parameters used are $\alpha=1, \sigma=1, X_{0}-\theta=2$, after the tree height $U_{n}$ was scaled to 1 . Right: The species tree disregarding the trait values supplied with the notation for the interspeciation times. For the pair of tips $(2,3)$ the time $\tau^{(n)}$ to their most recent common ancestor is marked on the time axis (starting at present and going back to the time of origin).

A detailed description of the evolutionary model along with our main results are presented in Section 2. Section 3 contains new formulae for the Laplace transforms of important characteristics of the conditioned Yule species tree: the time to origin $U_{n}$ and the time $\tau^{(n)}$ to the most recent common ancestor for a pair of two species chosen at random out of $n$ extant species. In Section 4 we calculate the interspecies correlation coefficient for the YuleOrnstein-Uhlenbeck model and Section 5 contains the proof of our limit theorems. In Section 6 we establish the consistency of the stationary variance estimator, which is needed for our confidence interval formulae, cf. [20] where the residual sum of squares was suggested to estimate the stationary variance. In Appendix A we calculate all the joint moments of $U_{n}$ and $\tau^{(n)}$.

Our main result, Theorem 1, should be compared with the limit theorems obtained in [1] and [2]. They also revealed three asymptotic regimes in a related, though different setting, dealing with a branching Ornstein-Uhlenbeck process. In their case the time of observation is deterministic and the number of the tree tips is random, while in our case the observation time is random and the number of the tips is deterministic. Although it is possible (with some effort) to deduce our results from [1] and [2], our proof provides a much more elementary derivation. We believe that our approach will be useful in addressing other biologically relevant issues such as the formulae for the higher moments given in Appendix A. Another similar limit theorem, but one conditional on the sequence of species trees generated by different mechanisms, was derived in [5].

\section{The model and main results}

This paper deals with what we call the Yule-Ornstein-Uhlenbeck model which is characterized by four parameters $\left(X_{0}, \alpha, \sigma, \theta\right)$ and consists of two ingredients.

(i) The species tree connecting $n$ extant species is modeled by the pure-birth Yule process [44] with a unit speciation rate $\lambda=1$ and conditioned on having $n$ tips [19]. 
(ii) The observed trait values $\left(X_{1}^{(n)}, \ldots, X_{n}^{(n)}\right)$ on the tips of the tree evolved from the ancestral state $X_{0}$ following the Ornstein-Uhlenbeck process with parameters $(\alpha, \sigma, \theta)$.

Definition 1. Let $\left(T_{1}, \ldots, T_{n}\right)$ be independent exponential random variables with parameters $(1, \ldots, n)$. We define the Yule $n$-tree as a random tree with $n$ tips that is constructed using a bottom-up algorithm based on the following two simple rules.

(i) During the time period $T_{k}$ the tree has $k$ branches.

(ii) For $k \in[2, n]$ the reduction from $k$ to $k-1$ branches occurs as two randomly chosen branches coalesce into one branch.

The height of the Yule $n$-tree is now $U_{n}=T_{1}+\cdots+T_{n}$.

As shown in [19], this definition corresponds to the standard Yule tree conditioned on having $n$ tips at the moment of observation, assuming that the time to the origin has the improper uniform prior.

Following [11] and [20], we model trait evolution along a lineage using the OrnsteinUhlenbeck process $X(t)$ given by the stochastic differential equation

$$
\mathrm{d} X(t)=-\alpha(X(t)-\theta) \mathrm{d} t+\sigma \mathrm{d} B(t), \quad X(0)=X_{0} .
$$

Here $\alpha>0$ is the adaptation rate, $\theta$ is the optimal trait value, $\sigma^{2}$ is the noise variance, and $B(t)$ is the standard Wiener process. The distribution of $X(t)$ is normal with

$$
\mathbb{E}[X(t)]=\theta+\mathrm{e}^{-\alpha t}\left(X_{0}-\theta\right), \quad \operatorname{var}[X(t)]=\frac{\sigma^{2}}{2 \alpha}\left(1-\mathrm{e}^{-2 \alpha t}\right),
$$

implying that $X(t)$ looses the effect of the ancestral state $X_{0}$ at an exponential rate. In the long run the Ornstein-Uhlenbeck process acquires a stationary normal distribution with mean $\theta$ and variance $\sigma^{2} / 2 \alpha$.

We propose asymptotic confidence interval formulae for the optimal value $\theta$, which take into account phylogenetic uncertainty. To this end we study properties of the sample mean and sample variance

$$
\bar{X}_{n}=\frac{X_{1}^{(n)}+\cdots+X_{n}^{(n)}}{n}, \quad S_{n}^{2}=\frac{1}{n-1} \sum_{i=1}^{n}\left(X_{i}^{(n)}-\bar{X}_{n}\right)^{2} .
$$

Using the properties of the Yule-Ornstein-Uhlenbeck model we find explicit expressions for $\mathbb{E}\left[\bar{X}_{n}\right], \operatorname{var}\left[\bar{X}_{n}\right], \mathbb{E}\left[S_{n}^{2}\right]$, study the asymptotics of $\operatorname{var}\left[S_{n}^{2}\right]$, and prove the following limit theorem revealing three different asymptotic regimes.

Theorem 1. Let $\delta=\left(X_{0}-\theta\right) / \sqrt{\sigma^{2} / 2 \alpha}$ be a normalized difference between the ancestral and optimal values. Consider the normalized sample mean $\bar{Y}_{n}=\left(\bar{X}_{n}-\theta\right) / \sqrt{\sigma^{2} / 2 \alpha}$ of the YuleOrnstein-Uhlenbeck process with $\bar{Y}_{0}=\delta$. As $n \rightarrow \infty$ the process $\bar{Y}_{n}$ has the following limit behavior.

(i) If $\alpha>\frac{1}{2}$ then $(\sqrt{n}) \bar{Y}_{n}$ is asymptotically normally distributed with 0 mean and variance $(2 \alpha+1) /(2 \alpha-1)$.

(ii) If $\alpha=\frac{1}{2}$ then $(\sqrt{n / \ln n}) \bar{Y}_{n}$ is asymptotically normally distributed with 0 mean and variance 2 . 
(iii) If $\alpha<\frac{1}{2}$ then $n^{\alpha} \bar{Y}_{n}$ converges almost surely (a.s.) and in $L^{2}$ to a random variable $Y_{\alpha, \delta}$ with $\mathbb{E}\left[Y_{\alpha, \delta}\right]=\delta \Gamma(1+\alpha)$ and $\mathbb{E}\left[Y_{\alpha, \delta}^{2}\right]=\left(\delta^{2}+4 \alpha /(1-2 \alpha)\right) \Gamma(1+2 \alpha)$.

Let $z_{x}$ be the $x$-quantile of the standard normal distribution, and $q_{x}$ be the $x$-quantile of the limit $Y_{\alpha, \delta}$. Denote by $S_{n}$ the sample standard deviation defined as the square root of $S_{n}^{2}$. As it will be shown in Section 6, the sample variance $S_{n}^{2}$ is a consistent estimator of $\sigma^{2} / 2 \alpha$. This fact together with Theorem 1 allows us to state the following three approximate $(1-x)$-level confidence intervals for $\theta$ assuming that we know the value of $\alpha$ :

$$
\begin{gathered}
\bar{X}_{n} \pm z_{1-x / 2} S_{n} \frac{K_{\alpha}}{\sqrt{n}}, \quad K_{\alpha}=\sqrt{\frac{2 \alpha+1}{2 \alpha-1}} \text { for } \alpha>\frac{1}{2} \\
\bar{X}_{n} \pm z_{1-x / 2} S_{n} \frac{\sqrt{2 \ln n}}{\sqrt{n}} \text { for } \alpha=\frac{1}{2} \\
\left(\bar{X}_{n}-q_{x / 2} S_{n} n^{-\alpha}, \bar{X}_{n}+q_{1-x / 2} S_{n} n^{-\alpha}\right) \text { for } \alpha<\frac{1}{2}
\end{gathered}
$$

Notably, the first of these confidence intervals differs from the classical confidence interval for the mean $\left(\bar{X}_{n} \pm z_{1-x / 2} S_{n} / \sqrt{n}\right)$ just by a factor $K_{\alpha}$. The latter is larger than 1 , as it should be, in view of a positive correlation among the sample observations. The correction factor $K_{\alpha}$ becomes negligible in the case of a very strong adaptation, $\alpha \gg 1$, when the dependence due to common ancestry can be neglected.

Remark 1. Observe that our standing assumption $\lambda=1$ (see Definition 1) of having one speciation event per unit of time causes no loss of generality. To incorporate an arbitrary speciation rate $\lambda$ one has to replace in our formulae parameters $\alpha$ and $\sigma^{2}$ by $\alpha / \lambda$ and $\sigma^{2} / \lambda$. This transformation corresponds to the time scaling by factor $\lambda$ in (1), it changes neither the optimal value $\theta$ nor the stationary variance $\sigma^{2} /(2 \alpha)$.

\section{Sampling $\boldsymbol{m}$ leaves from the Yule $\boldsymbol{n}$-tree}

Here we consider the Yule $n$-tree (see Definition 1 ) and study some properties of its subtree joining $m$ randomly (without replacement) chosen tips, where $m \in[2, n]$. In particular, we compute the joint Laplace transform of the height of the Yule $n$-tree $U_{n}=T_{1}+\cdots+T_{n}$ and $\tau^{(n)}$, the height of the most recent common ancestor for two randomly sampled tips; see Figure 1. For other results concerning the distribution of $\tau^{(n)}$ and $U_{n}$; see also [18], [19], [29], [35], [37], [38], [40], and [41].

Lemma 1. Consider a random $m$-subtree of the conditioned Yule $n$-tree. It has $m-1$ bifurcating events. Let $K_{1}^{(n, m)}<\cdots<K_{m-1}^{(n, m)}$ be the consecutive numbers of the bifurcation events in the Yule $n$-tree (counted from the root toward the leaves) corresponding to the $m-1$ bifurcating events of the $m$-subtree. Put $K_{0}^{(n, m)}=0$ and $K_{m}^{(n, m)}=n$. The sequence $\left(K_{m}^{(n, m)}, \ldots, K_{0}^{(n, m)}\right)$ forms a time-inhomogeneous Markov chain with transition probabilities

$$
\mathbb{P}\left(K_{j-1}^{(n, m)}=k \mid K_{j}^{(n, m)}=i\right)=p_{i k}^{(j)}, \quad 1 \leq j<k<i \leq n,
$$

where $p_{i, 0}^{(1)}=1$ for all $i \geq 1$, and

$$
p_{i k}^{(j)}=\frac{j(j-1)}{i k} \prod_{l=k+1}^{i-1} \frac{(l+1-j)(l+j)}{l^{2}}, \quad j=2, \ldots, m .
$$


Proof. Tracing the lineages of $m$ randomly sampled tips of the Yule $n$-tree towards the root, the first coalescent event can be viewed as the success in a sequence of independent Bernoulli trials. This argument leads to

$$
\mathbb{P}\left(K_{m-1}^{(n, m)}=k \mid K_{m}^{(n, m)}=n\right)=\left(1-\frac{\left(\begin{array}{c}
m \\
2
\end{array}\right)}{\left(\begin{array}{c}
n \\
2
\end{array}\right)}\right) \cdots\left(1-\frac{\left(\begin{array}{c}
m \\
2
\end{array}\right)}{\left(\begin{array}{c}
k+2 \\
2
\end{array}\right)}\right) \frac{\left(\begin{array}{c}
m \\
2
\end{array}\right)}{\left(\begin{array}{c}
k+1 \\
2
\end{array}\right)}
$$

resulting in the desired expression for $p_{n k}^{(m)}$ with $m-1 \leq k \leq n-1$, cf. [38]. The transition probabilities $p_{i k}^{(j)}$ for $j=2, \ldots, m-1$ are obtained similarly.

Lemma 2. Consider the interbifurcation times for the $m$-subtree of the Yule $n$-tree

$$
\chi_{j}^{(n, m)}=T_{K_{j-1}^{(n, m)}+1}+\cdots+T_{K_{j}^{(n, m)}}, \quad j=1, \ldots, m,
$$

so that $U_{n}=\chi_{1}^{(n, m)}+\cdots+\chi_{m}^{(n, m)}$ for any $m \leq n$, and $\tau^{(n)}=\chi_{2}^{(n, 2)}$. Then for $x_{j}>-1$, we have

$$
\mathbb{E}\left[\exp \left\{-\sum_{j=1}^{m} x_{j} \chi_{j}^{(n, m)}\right\}\right]=\sum_{k_{1}=1}^{n-1} \sum_{k_{2}=k_{1}+1}^{n-1} \cdots \sum_{k_{m-1}=k_{m-2}+1}^{n-1} \prod_{j=1}^{m} p_{k_{j}, k_{j-1}}^{(j)} \frac{b_{k_{j}, x_{j-1}}}{b_{k_{j-1}, x_{j-1}}}
$$

where $k_{m}=n, k_{0}=0$, and

$$
b_{n, x}=\frac{1}{1+x} \frac{2}{2+x} \ldots \frac{n}{n+x}=\frac{\Gamma(n+1) \Gamma(x+1)}{\Gamma(n+x+1)}, \quad x>-1 .
$$

Proof. The Laplace transform of the sum of independent exponentials:

$$
\begin{aligned}
& \mathbb{E}\left[\exp \left\{-x_{1} \chi_{1}^{(n, m)}-\cdots-x_{m} \chi_{m}^{(n, m)}\right\} \mid\left(K_{m-1}^{(n, m)}, \ldots, K_{1}^{(n, m)}\right)=\left(k_{m-1}, \ldots, k_{1}\right)\right] \\
& \quad=\prod_{j=1}^{m} \frac{k_{j-1}+1}{x_{j-1}+k_{j-1}+1} \cdots \frac{k_{j}}{x_{j-1}+k_{j}}
\end{aligned}
$$

together with Lemma 1 implies the stated equality.

Lemma 3. The joint Laplace transform of the height of the Yule $n$-tree and the height of the most recent common ancestor for two randomly sampled tips is given by

$$
\mathbb{E}\left[\mathrm{e}^{-x U_{n}-y \tau^{(n)}}\right]=\frac{2(n+1) b_{n, x+y}}{(n-1)} \sum_{k=1}^{n-1} \frac{b_{k, x}}{(k+2)(k+1) b_{k, x+y}} .
$$

In particular,

$$
\mathbb{E}\left[\mathrm{e}^{-x U_{n}}\right]=b_{n, x}, \quad \operatorname{var}\left[\mathrm{e}^{-x U_{n}}\right]=b_{n, 2 x}-b_{n, x}^{2},
$$

and denoting the harmonic number $h_{n}:=1+\frac{1}{2}+\cdots+1 / n$,

$$
\mathbb{E}\left[\mathrm{e}^{-y \tau^{(n)}}\right]= \begin{cases}\frac{2-(n+1)(y+1) b_{n, y}}{(n-1)(y-1)} & \text { for } y \neq 1, \\ \frac{2}{n-1}\left(h_{n}-1\right)-\frac{1}{n+1} & \text { for } y=1\end{cases}
$$


Proof. Turning to Lemma 1 with $m=2$, we obtain

$$
p_{n, k}^{(2)}=\frac{2}{n k} \prod_{i=k+1}^{n-1} \frac{(i-1)(i+2)}{i^{2}}=\frac{2(n+1)}{(n-1)(k+2)(k+1)}, \quad k=1, \ldots, n-1,
$$

and according to Lemma 2 ,

$$
\mathbb{E}\left[\mathrm{e}^{-x\left(U_{n}-\tau^{(n)}\right)-y \tau^{(n)}}\right]=\sum_{k=1}^{n-1} p_{n, k}^{(2)} \frac{1}{x+1} \cdots \frac{k}{x+k} \frac{k+1}{y+k+1} \cdots \frac{n}{y+n}
$$

resulting in the desired equation. This implies the main formula claimed by Lemma 3 giving $\mathbb{E}\left[\mathrm{e}^{-x U_{n}}\right]=b_{n, x}$ after putting $y=0$. With $x=0$, we obtain

$$
\mathbb{E}\left[\mathrm{e}^{-y \tau^{(n)}}\right]=\frac{2(n+1) !}{(n-1) \Gamma(y+n+1)} \sum_{k=1}^{n-1} \frac{\Gamma(k+1+y)}{\Gamma(k+3)} .
$$

When $y=1$ this directly becomes

$$
\mathbb{E}\left[\mathrm{e}^{-\tau^{(n)}}\right]=\frac{2}{n-1}\left(h_{n}-1\right)-\frac{1}{n+1} .
$$

In the $y \neq 1$ case we use the relation (easily verified by induction when $z \neq y$ )

$$
\sum_{k=1}^{n-1} \frac{\Gamma(k+y)}{\Gamma(k+z+1)}=\frac{\Gamma(n+z) \Gamma(y+1)-\Gamma(z+1) \Gamma(n+y)}{\Gamma(z+1) \Gamma(n+z)(z-y)}
$$

to derive

$$
\mathbb{E}\left[\mathrm{e}^{-y \tau^{(n)}}\right]=\frac{2 \Gamma(n+1+y)-\Gamma(n+2) \Gamma(y+2)}{(n-1) \Gamma(y+n+1)(y-1)}=\frac{2-(n+1)(y+1) b_{n, y}}{(n-1)(y-1)} .
$$

Lemma 4. As $n \rightarrow \infty$ for positive $x$ and $y$, we have the following asymptotic results:

$$
\begin{aligned}
\mathbb{E}\left[\mathrm{e}^{-x U_{n}}\right] & \sim \Gamma(x+1) n^{-x}, \\
\mathbb{E}\left[\mathrm{e}^{-y \tau^{(n)}}\right] & \sim \begin{cases}\frac{1+y}{1-y} \Gamma(y+1) n^{-y} & \text { if } 0<y<1, \\
2 n^{-1} \ln n & \text { if } y=1, \\
\frac{2}{y-1} n^{-1} & \text { if } y>1,\end{cases} \\
\mathbb{E}\left[\mathrm{e}^{\left.-x U_{n}-y \tau^{(n)}\right]}\right. & \sim \begin{cases}C_{x, y} n^{-x-y} & \text { if } 0<y<1, \\
2 \Gamma(x+1) n^{-x-1} \ln n & \text { if } y=1, \\
\frac{2 \Gamma(x+1)}{y-1} n^{-x-1} & \text { if } y>1,\end{cases}
\end{aligned}
$$

where

$$
C_{x, y}=2 \Gamma(x+y+1) \sum_{k=1}^{\infty} \frac{b_{k, x}}{(k+2)(k+1) b_{k, x+y}}
$$


Proof. The stated results are obtained from Lemma 3 using the fact that for large $n$, we have $b_{n, x} \sim \Gamma(x+1) n^{-x}$ and, furthermore,

$$
\frac{1-(n+1) b_{n, x}}{x-1} \longrightarrow h_{n}-1 \quad \text { as } x \rightarrow 1, \quad x^{-1}\left(1-b_{n, x}\right) \longrightarrow h_{n} \quad \text { as } x \rightarrow 0 .
$$

These three relations will often be used tacitly in what follows.

\section{Interspecies correlation}

Denote by $y_{n}$ the $\sigma$-algebra containing all information on the Yule $n$-tree. The scaled trait values $Y_{i}^{(n)}:=\left(X_{i}^{(n)}-\theta\right) /(\sigma / \sqrt{2 \alpha})$, in view of $(2)$, are conditionally normal with

$$
\mathbb{E}\left[Y_{i}^{(n)} \mid y_{n}\right]=\delta \mathrm{e}^{-\alpha U_{n}}, \quad \operatorname{var}\left[Y_{i}^{(n)} \mid y_{n}\right]=1-\mathrm{e}^{-2 \alpha U_{n}},
$$

which together with the results from Section 3 entails

$$
\mathbb{E}\left[Y_{i}^{(n)}\right]=\delta b_{n, \alpha}, \quad \operatorname{var}\left[Y_{i}^{(n)}\right]=1-\delta^{2} b_{n, \alpha}^{2}+\left(\delta^{2}-1\right) b_{n, 2 \alpha}
$$

Lemma 5. In the framework of the Yule-Ornstein-Uhlenbeck model, for an arbitrary pair of traits, we have

$$
\operatorname{cov}\left[Y_{i}^{(n)}, Y_{j}^{(n)} \mid y_{n}\right]=\mathrm{e}^{-2 \alpha \tau_{i j}^{(n)}}-\mathrm{e}^{-2 \alpha U_{n}},
$$

where $\tau_{i j}^{(n)}$ is the backward time to the most recent common ancestor of the tips $(i, j)$.

Proof. Denote by $Y_{i j}^{(n)}$ the normalized trait value of the most recent common ancestor of the tips $(i, j)$. Let $y_{i j}^{(n)}$ stand for the $\sigma$-algebra generated by $\left(y_{n}, Y_{i j}^{(n)}\right)$, then using (2), we obtain

$$
\operatorname{cov}\left[Y_{i}^{(n)}, Y_{j}^{(n)} \mid y_{i j}^{(n)}\right]=0, \quad \mathbb{E}\left[Y_{i} \mid y_{i j}^{(n)}\right]=\mathbb{E}\left[Y_{j} \mid y_{i j}^{(n)}\right]=\mathrm{e}^{-\alpha \tau_{i j}^{(n)}} Y_{i j}^{(n)},
$$

implying the statement of this lemma:

$$
\operatorname{cov}\left[Y_{i}^{(n)}, Y_{j}^{(n)} \mid y_{n}\right]=\operatorname{var}\left[\mathrm{e}^{-\alpha \tau_{i j}^{(n)}} Y_{i j}^{(n)} \mid y_{n}\right]=\mathrm{e}^{-2 \alpha \tau_{i j}^{(n)}}-\mathrm{e}^{-2 \alpha U_{n}}
$$

Lemma 6. Consider the interspecies correlation coefficient, the unconditioned correlation between two randomly sampled trait values

$$
\rho_{n}=\frac{1}{n(n-1)} \sum_{i} \sum_{j \neq j} \frac{\operatorname{cov}\left[X_{i}^{(n)}, X_{j}^{(n)}\right]}{\operatorname{var}\left[X_{1}^{(n)}\right]}=\frac{1}{n(n-1)} \sum_{i} \sum_{j \neq j} \frac{\operatorname{cov}\left[Y_{i}^{(n)}, Y_{j}^{(n)}\right]}{\operatorname{var}\left[Y_{1}^{(n)}\right]}
$$

If $\alpha \neq \frac{1}{2}$ then

$$
\rho_{n}=1-\frac{2 \alpha(n-1)+(n+1)\left((1+2 \alpha) b_{n, 2 \alpha}-1\right)}{(n-1)(2 \alpha-1)\left(1+\left(\delta^{2}-1\right) b_{n, 2 \alpha}-\delta^{2} b_{n, \alpha}^{2}\right)},
$$

and in the case of $\alpha=\frac{1}{2}$,

$$
\rho_{n}=1-\frac{n+1}{n-1} \frac{n+2-2 h_{n}}{n+\delta^{2}\left(1-(n+1) b_{n, 0.5}^{2}\right)} .
$$


Proof. According to Lemma 5, we have

$$
\frac{2}{n(n-1)} \sum_{i<j} \operatorname{cov}\left[Y_{i}^{(n)}, Y_{j}^{(n)}\right]=\mathbb{E}\left[\mathrm{e}^{-2 \alpha \tau^{(n)}}-\mathrm{e}^{-2 \alpha U_{n}}\right]+\delta^{2} \operatorname{var}\left[\mathrm{e}^{-\alpha U_{n}}\right]
$$

leading to

$$
\rho_{n}=1-\frac{1-\mathbb{E}\left[\mathrm{e}^{-2 \alpha \tau^{(n)}}\right]}{1-\mathbb{E}\left[\mathrm{e}^{-2 \alpha U_{n}}\right]+\delta^{2} \operatorname{var}\left[\mathrm{e}^{-\alpha U_{n}}\right]} .
$$

Applying the results of Section 3 we arrive at the asserted relations for $\rho_{n}$. Observe that asymptotically as $n \rightarrow \infty$ the interspecies correlation coefficient decays to 0 as

$$
\rho_{n} \sim \begin{cases}\frac{2}{1-2 \alpha} \Gamma(1+2 \alpha)+\delta^{2}\left(\Gamma(1+2 \alpha)-\Gamma^{2}(\alpha+1)\right) n^{-2 \alpha}, & 0<\alpha<\frac{1}{2}, \\ 2 n^{-1} \ln n, & \alpha=\frac{1}{2}, \\ \frac{2}{2 \alpha-1} n^{-1} & \alpha>\frac{1}{2} .\end{cases}
$$

Lemma 7. Consider the sample mean $\bar{Y}_{n}=n^{-1}\left(Y_{1}^{(n)}+\cdots+Y_{n}^{(n)}\right)$ and the sample variance

$$
D_{n}^{2}=\frac{1}{n-1} \sum_{i=1}^{n}\left(Y_{i}^{(n)}-\bar{Y}_{n}\right)^{2}
$$

of the scaled trait values. For all $\alpha>0$, we have $\mathbb{E}\left[\bar{Y}_{n}\right]=\delta b_{n, \alpha}$. For $\alpha \neq \frac{1}{2}$,

$$
\begin{gathered}
\operatorname{var}\left[\bar{Y}_{n}\right]=\frac{1+2 \alpha-(4 \alpha n+1+2 \alpha) b_{n, 2 \alpha}}{(2 \alpha-1) n}+\delta^{2}\left(b_{n, 2 \alpha}-b_{n, \alpha}^{2}\right), \\
\mathbb{E}\left[D_{n}^{2}\right]=1+\frac{(1+2 \alpha)(n+1) b_{n, 2 \alpha}-2}{(2 \alpha-1)(n-1)},
\end{gathered}
$$

and in the singular $\alpha=\frac{1}{2}$ case,

$$
\operatorname{var}\left[\bar{Y}_{n}\right]=\frac{2\left(h_{n}-1\right)}{n}+\frac{\delta^{2}-1}{n+1}-\delta^{2} b_{n, 0.5}^{2}, \quad \mathbb{E}\left[D_{n}^{2}\right]=\frac{n-2 h_{n}}{n-1} .
$$

Proof. Obviously, $\mathbb{E}\left[\bar{Y}_{n}\right]=\mathbb{E}\left[Y_{i}^{(n)}\right]=\delta b_{n, \alpha}$. To prove the other assertions we turn to [35], where the concept of interspecies correlation was originally introduced. It was shown that the variance of the sample average and the expectation of the sample variance can be compactly expressed as

$$
\operatorname{var}\left[\bar{X}_{n}\right]=\left(\frac{1}{n}+\frac{n-1}{n} \rho_{n}\right) \operatorname{var}\left[X_{1}^{(n)}\right], \quad \mathbb{E}\left[S_{n}^{2}\right]=\left(1-\rho_{n}\right) \operatorname{var}\left[X_{1}^{(n)}\right] .
$$

Since

$$
\operatorname{var}\left[Y_{1}^{(n)}\right]=\left(\frac{2 \alpha}{\sigma^{2}}\right) \operatorname{var}\left[X_{1}^{(n)}\right], \quad \operatorname{var}\left[\bar{Y}_{n}\right]=\left(\frac{2 \alpha}{\sigma^{2}}\right) \operatorname{var}\left[\bar{X}_{n}\right], \quad \mathbb{E}\left[D_{n}^{2}\right]=\left(\frac{2 \alpha}{\sigma^{2}}\right) \mathbb{E}\left[S_{n}^{2}\right],
$$

it remains to combine Lemma 6 with the known expression for $\operatorname{var}\left[Y_{1}^{(n)}\right]$. 
A more direct proof of Lemma 7 can be obtained using the following result on conditional expectations.

Lemma 8. We have

$$
\begin{gathered}
\mathbb{E}\left[\bar{Y}_{n} \mid y_{n}\right]=\delta \mathrm{e}^{-\alpha U_{n}}, \\
\mathbb{E}\left[\bar{Y}_{n}^{2} \mid y_{n}\right]=\frac{1}{n}+\left(1-\frac{1}{n}\right) \mathbb{E}\left[\mathrm{e}^{-2 \alpha \tau^{(n)}} \mid y_{n}\right]-\mathrm{e}^{-2 \alpha U_{n}}+\delta^{2} \mathrm{e}^{-2 \alpha U_{n}}, \\
\operatorname{var}\left[\bar{Y}_{n} \mid y_{n}\right]=\frac{1}{n}+\left(1-\frac{1}{n}\right) \mathbb{E}\left[\mathrm{e}^{-2 \alpha \tau^{(n)}} \mid y_{n}\right]-\mathrm{e}^{-2 \alpha U_{n}} .
\end{gathered}
$$

Proof. The main assertion follows from

$$
\begin{aligned}
\operatorname{var}\left[Y_{1}^{(n)}+\cdots+Y_{n}^{(n)} \mid y_{n}\right] & =n\left(1-\mathrm{e}^{-2 \alpha U_{n}}\right)+2 \sum_{i<j}\left(\mathrm{e}^{-2 \alpha \tau_{i j}^{(n)}}-\mathrm{e}^{-2 \alpha U_{n}}\right) \\
& =n-n^{2} \mathrm{e}^{-2 \alpha U_{n}}+n(n-1) \mathbb{E}\left[\mathrm{e}^{-2 \alpha \tau^{(n)}} \mid y_{n}\right] .
\end{aligned}
$$

\section{Proof of Theorem 1}

Lemma 9. Put $V_{n}^{(x)}:=b_{n, x}^{-1} \mathrm{e}^{-x U_{n}}$ with $\mathbb{E}\left[V_{n}^{(x)}\right]=1$. For any $x>-1$, the sequence $\left\{V_{n}^{(x)}, y_{n}\right\}_{n \geq 0}$ forms a martingale converging a.s. and in $L^{2}$. Moreover, $\left(U_{n}-\log n\right)$ converges in distribution to a random variable having the standard Gumbel distribution.

Proof. The martingale property is obvious:

$$
\mathbb{E}\left[V_{n+1}^{(x)} \mid y_{n}\right]=\frac{\mathrm{e}^{-x U_{n}} \mathbb{E}\left[\mathrm{e}^{-x T_{n+1}}\right]}{b_{n+1, x}}=\frac{\mathrm{e}^{-x U_{n}}}{b_{n, x}}=V_{n}^{(x)}
$$

Since the second moments

$$
\mathbb{E}\left[\left(V_{n}^{(x)}\right)^{2}\right]=\frac{\mathbb{E}\left[\mathrm{e}^{-2 x U_{n}}\right]}{b_{n, x}^{2}}=\frac{b_{n, 2 x}}{\left(b_{n, x}\right)^{2}}
$$

are uniformly bounded over $n$, we conclude that $V_{n}^{(x)} \rightarrow V^{(x)}$ a.s. and in $L^{2}$ with $\mathbb{E}\left[V^{(x)}\right]=1$. It follows that $\mathbb{E}\left[V_{n}^{(x)}\right] \rightarrow 1$ and, therefore, $\mathbb{E}\left[\mathrm{e}^{-x\left(U_{n}-\log n\right)}\right] \rightarrow \Gamma(x+1)$. The latter is a convergence of Laplace transforms confirming the stated convergence in distribution.

Observe that the Gumbel limit for $U_{n}-\log n$ can be obtained using the classical extreme value theory, in view of the representation

$$
U_{n} \stackrel{\mathrm{D}}{=} \sum_{i=1}^{n} \frac{1}{i} E_{i} \stackrel{\mathrm{D}}{=} \max \left(E_{1}, \ldots, E_{n}\right)
$$

in terms of independent exponentials with parameter 1 , where ' $=$ ' denotes equality in distribution. Note also that $U_{n+1} / 2$ has the same distribution as the total branch length of Kingman's $n$-coalescent.

Lemma 10. Denote by $\mathcal{F}_{n}$ the $\sigma$-algebra containing information on the Yule $n$-tree realization as well as the corresponding information on the evolution of trait values. Set

$$
H_{n}:=(n+1) \mathrm{e}^{(\alpha-1) U_{n}} \bar{Y}_{n}, \quad n \geq 0 .
$$

The sequence $\left\{H_{n}, \mathcal{F}_{n}\right\}_{n \geq 0}$ forms a martingale with $\mathbb{E}\left[H_{n}\right]=H_{0}=\delta$. 
Proof. Note that

$$
\begin{aligned}
\mathbb{E}\left[\mathrm{e}^{(\alpha-1) T_{n+1}} \sum_{i=1}^{n+1} Y_{i}^{(n+1)} \mid \mathcal{F}_{n}\right] & =\mathbb{E}\left[\mathrm{e}^{-T_{n+1}}\right]\left(\sum_{i=1}^{n} Y_{i}^{(n)}+n^{-1} \sum_{j=1}^{n} Y_{j}^{(n)}\right) \\
& =\frac{n+1}{n+2} \frac{n+1}{n} \sum_{i=1}^{n} Y_{i}^{(n)} \\
& =\frac{(n+1)^{2}}{n+2} \bar{Y}_{n} .
\end{aligned}
$$

Hence,

$$
\mathbb{E}\left[H_{n+1} \mid \mathcal{F}_{n}\right]=\frac{n+2}{n+1} \mathrm{e}^{(\alpha-1) U_{n}} \mathbb{E}\left[\mathrm{e}^{(\alpha-1) T_{n+1}} \sum_{i=1}^{n+1} Y_{i}^{(n+1)} \mid \mathcal{F}_{n}\right]=H_{n} .
$$

Lemma 11. For all positive $\alpha$, we have $\operatorname{var}\left[\mathbb{E}\left[\mathrm{e}^{-2 \alpha \tau^{(n)}} \mid y_{n}\right]\right]=O\left(n^{-3}\right)$ as $n \rightarrow \infty$.

Proof. For a given realization of the Yule $n$-tree, we denote by $\tau_{1}^{(n)}$ and $\tau_{2}^{(n)}$ two independent versions of $\tau^{(n)}$ corresponding to two independent choices of pairs of tips out of $n$ available. We have

$$
\mathbb{E}\left[\left(\mathbb{E}\left[\mathrm{e}^{-2 \alpha \tau^{(n)}} \mid y_{n}\right]\right)^{2}\right]=\mathbb{E}\left[\mathbb{E}\left[\mathrm{e}^{-2 \alpha\left(\tau_{1}^{(n)}+\tau_{2}^{(n)}\right)} \mid y_{n}\right]\right]=\mathbb{E}\left[\mathrm{e}^{-2 \alpha\left(\tau_{1}^{(n)}+\tau_{2}^{(n)}\right)}\right] .
$$

Writing

$$
\pi_{n, k}:=p_{n, k}^{(2)}, \quad f(a, k, n)=\frac{k+1}{a+k+1} \cdots \frac{n}{a+n}
$$

and using the ideas of Section 3, we obtain

$$
\mathbb{E}\left[\left(\mathbb{E}\left[\mathrm{e}^{-2 \alpha \tau^{(n)}} \mid y_{n}\right]\right)^{2}\right]=\sum_{k=1}^{n-1} f_{4 \alpha}(k, n) \pi_{n, k}^{2}+2 \sum_{k_{1}<k_{2}} f_{2 \alpha}\left(k_{1}, k_{2}\right) f_{4 \alpha}\left(k_{2}, n\right) \pi_{n, k_{1}} \pi_{n, k_{2}} .
$$

On the other hand,

$$
\left(\mathbb{E}\left[\mathrm{e}^{-2 \alpha \tau^{(n)}}\right]\right)^{2}=\left(\sum_{k_{1}} f_{2 \alpha}\left(k_{1}, n\right) \pi_{n, k_{1}}\right)\left(\sum_{k_{2}} f_{2 \alpha}\left(k_{2}, n\right) \pi_{n, k_{2}}\right) .
$$

Taking the difference between the last two expressions, we have

$$
\begin{aligned}
\operatorname{var}\left[\mathbb{E}\left[\mathrm{e}^{-2 \alpha \tau^{(n)}} \mid y_{n}\right]\right] \\
=\sum_{k}\left(f_{4 \alpha}(k, n)-f_{2 \alpha}(k, n)^{2}\right) \pi_{n, k}^{2} \\
\quad+2 \sum_{k_{1}=1}^{n-1} \sum_{k_{2}=k_{1}+1}^{n-1} f_{2 \alpha}\left(k_{1}, k_{2}\right)\left(f_{4 \alpha}\left(k_{2}, n\right)-f_{2 \alpha}\left(k_{2}, n\right)^{2}\right) \pi_{n, k_{1}} \pi_{n, k_{2}} .
\end{aligned}
$$

Using the simple equality

$$
a_{1} \cdots a_{n}-b_{1} \cdots b_{n}=\sum_{i=1}^{n} b_{1} \cdots b_{i-1}\left(a_{i}-b_{i}\right) a_{i+1} \cdots a_{n}
$$


we see that it suffices to prove that

$$
\sum_{k=1}^{n-1} A_{n, k} \pi_{n, k}^{2}=O\left(n^{-4}\right), \quad \sum_{k_{1}=1}^{n-1} \sum_{k_{2}=k_{1}+1}^{n-1} f_{2 \alpha}\left(k_{1}, k_{2}\right) A_{n, k_{2}} \pi_{n, k_{1}} \pi_{n, k_{2}}=O\left(n^{-3}\right),
$$

where

$$
A_{n, k}:=\sum_{j=k+1}^{n-1} f_{2 \alpha}(k, j)^{2}\left(\frac{2 \alpha}{2 \alpha+j+1}\right)^{2} f_{4 \alpha}(j, n) .
$$

To verify these two asymptotic relations observe that

$$
A_{n, k}<\frac{k+1}{4 \alpha+k+1} \cdots \frac{n}{4 \alpha+n} \sum_{i=k+1}^{n} \frac{4 \alpha^{2}}{(2 \alpha+i)^{2}}<\frac{4 \alpha^{2} b_{n, 4 \alpha}}{b_{k, 4 \alpha}} \sum_{i=k+1}^{n} \frac{1}{i(i-1)}<\frac{4 \alpha^{2} b_{n, 4 \alpha}}{k b_{k, 4 \alpha}} .
$$

Since $\pi_{n, k}=2(n+1) /(n-1)(k+2)(k+1)$, it follows that

$$
\sum_{k=1}^{n-1} A_{n, k} \pi_{n, k}^{2}<c_{1} b_{n, 4 \alpha} \sum_{k=1}^{n-1} \frac{1}{k^{5} b_{k, 4 \alpha}}<c_{2} n^{-4 \alpha} \sum_{k=1}^{n} n^{4 \alpha-5}<c_{2} n^{-4}
$$

and

$$
\begin{aligned}
\sum_{k_{1}=1}^{n-1} \sum_{k_{2}=k_{1}+1}^{n-1} f_{2 \alpha}\left(k_{1}, k_{2}\right) A_{n, k_{2}} \pi_{n, k_{1}} \pi_{n, k_{2}} & <c_{3} b_{n, 4 \alpha} \sum_{k_{1}=1}^{n-1} \sum_{k_{2}=k_{1}+1}^{n-1} \frac{b_{k_{2}, 2 \alpha}}{b_{k_{1}, 2 \alpha} b_{k_{2}, 4 \alpha}} \frac{1}{k_{1}^{2} k_{2}^{3}} \\
& <c_{4} n^{-4 \alpha} \sum_{k_{2}=2}^{n} k_{2}^{2 \alpha-3} \sum_{k_{1}=1}^{k_{2}} k_{1}^{2 \alpha-2} \\
& <c_{4} n^{-4 \alpha} \sum_{k_{2}=2}^{n} k_{2}^{4 \alpha-4} \\
& <c_{4} n^{-3} .
\end{aligned}
$$

Proof of Theorem 1(i) and 1(ii). Let $\alpha>\frac{1}{2}$. To establish the stated normal approximation it is enough to prove the convergence in probability of the first two conditional moments

$$
\left(\mu_{n}, \sigma_{n}^{2}\right):=\left(\sqrt{n} \mathbb{E}\left[\bar{Y}_{n} \mid \mathcal{Y}_{n}\right], n \operatorname{var}\left[\bar{Y}_{n} \mid \mathcal{y}_{n}\right]\right) \stackrel{\mathbb{P}}{\rightarrow}\left(0, \frac{2 \alpha+1}{2 \alpha-1}\right), \quad n \rightarrow \infty,
$$

since then, due to the conditional normality of $\bar{Y}_{n}$, we will obtain the following convergence of characteristic functions:

$$
\mathbb{E}\left[\mathrm{e}^{\mathrm{i} \gamma \sqrt{n}\left(\bar{Y}_{n}\right)}\right]=\mathbb{E}\left[\mathrm{e}^{\mathrm{i} \mu_{n} \gamma-\sigma_{n}^{2} \gamma^{2} / 2}\right] \rightarrow \mathrm{e}^{-((2 \alpha+1) / 2(2 \alpha-1)) \gamma^{2}} .
$$

Now, due to Lemma 8 , we can write

$$
\left(\mu_{n}, \sigma_{n}^{2}\right)=\left(\sqrt{n} \delta \mathrm{e}^{-\alpha U_{n}}, 1+(n-1) \mathbb{E}\left[\mathrm{e}^{-2 \alpha \tau^{(n)}} \mid y_{n}\right]-n \mathrm{e}^{-2 \alpha U_{n}}\right) .
$$


Using (3) and (4), we see that

$$
\mathbb{E}\left[\sigma_{n}^{2}\right]=1-n b_{n, 2 \alpha}+\frac{2-(n+1)(2 \alpha+1) b_{n, 2 \alpha}}{2 \alpha-1} \rightarrow \frac{2 \alpha+1}{2 \alpha-1} .
$$

It remains to observe that on the one hand, according to Lemma 11,

$$
1+(n-1) \mathbb{E}\left[\mathrm{e}^{-2 \alpha \tau^{(n)}} \mid y_{n}\right] \stackrel{\mathbb{P}}{\rightarrow} \frac{2 \alpha+1}{2 \alpha-1},
$$

and on the other hand, $n \mathrm{e}^{-2 \alpha U_{n}} \stackrel{\mathbb{P}}{\rightarrow} 0$, implying that $\sigma_{n}^{2} \stackrel{\mathbb{P}}{\rightarrow}(2 \alpha+1) /(2 \alpha-1)$. This together with $\mu_{n} \rightarrow 0$ holding in $L^{2}$ and, therefore, in probability, entails $\left(\mu_{n}, \sigma_{n}^{2}\right) \stackrel{\mathbb{P}}{\rightarrow}(0,(2 \alpha+1) /(2 \alpha-$ 1)), finishing the proof of Theorem 1(i). Theorem 1(ii) is proven similarly.

Proof of Theorem 1(iii). Let $0<\alpha<\frac{1}{2}$. Turning to Lemma 10, observe that the martingale $H_{n}=(n+1) \mathrm{e}^{(\alpha-1) U_{n}} \bar{Y}_{n}$ has uniformly bounded second moments. Indeed, due to Lemma 8 ,

$$
\begin{aligned}
\mathbb{E}\left[H_{n}^{2}\right] & =(n+1)^{2} \mathbb{E}\left[\mathrm{e}^{2(\alpha-1) U_{n}} \mathbb{E}\left[\bar{Y}_{n}^{2} \mid y_{n}\right]\right] \\
& <c_{1} n \mathbb{E}\left[\mathrm{e}^{-2(1-\alpha) U_{n}}\right]+c_{2} n^{2} \mathbb{E}\left[\mathrm{e}^{-2(1-\alpha) U_{n}-2 \alpha \tau^{(n)}}\right]+c_{3} n^{2} \mathbb{E}\left[\mathrm{e}^{-2 \alpha U_{n}}\right] .
\end{aligned}
$$

Thus, according to Lemma 4 we have $\sup _{n} \mathbb{E}\left[H_{n}^{2}\right]<\infty$. Referring to the martingale $L^{2}$ convergence theorem, we conclude that $H_{n} \rightarrow H_{\infty}$ a.s. and in $L^{2}$. Due to Lemma 9, it follows that

$$
n^{\alpha} \bar{Y}_{n}=\frac{n^{\alpha} b_{n, \alpha-1}}{n+1} V_{n}^{(\alpha-1)} H_{n} \rightarrow V^{(\alpha-1)} H_{\infty}=: Y_{\alpha, \delta} \quad \text { a.s. and in } L^{2}
$$

Finally,

$$
\begin{aligned}
n^{\alpha} \mathbb{E}\left[\bar{Y}_{n}\right] & =\delta n^{\alpha} b_{n, \alpha} \rightarrow \delta \Gamma(1+\alpha) \\
n^{2 \alpha} \mathbb{E}\left[\bar{Y}_{n}^{2}\right] & =n^{2 \alpha-1}+n^{2 \alpha}\left(1-\frac{1}{n}\right) \mathbb{E}\left[\mathrm{e}^{-2 \alpha \tau^{(n)}}\right]+n^{2 \alpha}\left(\delta^{2}-1\right) \mathbb{E}\left[\mathrm{e}^{-2 \alpha U_{n}}\right] \\
& \rightarrow\left(\delta^{2}+\frac{4 \alpha}{1-2 \alpha}\right) \Gamma(1+2 \alpha) \quad \text { as } n \rightarrow \infty
\end{aligned}
$$

\section{Consistency of the sample variance}

Recall that $\mathbb{E}\left[S_{n}^{2}\right]=\left(\sigma^{2} / 2 \alpha\right) \mathbb{E}\left[D_{n}^{2}\right]$ and according to Lemma 7 , we have $\mathbb{E}\left[D_{n}^{2}\right] \rightarrow 1$. The aim of this section is to show that $\operatorname{var}\left[D_{n}^{2}\right] \rightarrow 0$ as $n \rightarrow \infty$ which is equivalent to $\mathbb{E}\left[D_{n}^{4}\right] \rightarrow 1$ in this regime. To this end we will need the following formula [9, Equation (13)] valid for any normally distributed vector $\left(Z_{1}, Z_{2}, Z_{3}, Z_{4}\right)$ with means $\left(m_{1}, m_{2}, m_{3}, m_{4}\right)$ and covariances $\operatorname{cov}\left[Z_{i}, Z_{j}\right]=c_{i j}$ :

$$
\operatorname{cov}\left[Z_{1} Z_{2}, Z_{3} Z_{4}\right]=m_{1} m_{3} c_{24}+m_{1} m_{4} c_{23}+m_{2} m_{3} c_{14}+m_{2} m_{4} c_{13}+c_{13} c_{24}+c_{14} c_{23}
$$

for the special case $m_{i}=m$ in order to calculate $\mathbb{E}\left[Z_{1} Z_{2} Z_{3} Z_{4}\right]$. Writing $Y_{i}$ instead of $Y_{i}^{(n)}$, we use the representation

$$
D_{n}^{2}=\frac{n}{n-1}\left(\frac{1}{n} \sum_{i=1}^{n} Y_{i}^{2}-\bar{Y}_{n}^{2}\right)=\frac{1}{n} \sum_{i} Y_{i}^{2}-\frac{2}{n(n-1)} \sum_{i} \sum_{j>i} Y_{i} Y_{j}
$$


to deduce that

$$
\begin{aligned}
\mathbb{E}\left[D_{n}^{4}\right]= & \frac{1}{n^{2}}\left(\sum_{i} \mathbb{E}\left[Y_{i}^{4}\right]+2 \sum_{i} \sum_{j>i} \mathbb{E}\left[Y_{i}^{2} Y_{j}^{2}\right]\right) \\
& -\frac{4}{n^{2}(n-1)}\left(\sum_{i} \sum_{j>i} \mathbb{E}\left[Y_{i}^{3} Y_{j}\right]+\sum_{i} \sum_{j>i} \mathbb{E}\left[Y_{i} Y_{j}^{3}\right]+\sum_{i} \sum_{j>i} \sum_{k \neq i, j} \mathbb{E}\left[Y_{i}^{2} Y_{j} Y_{k}\right]\right) \\
& +\frac{4}{n^{2}(n-1)^{2}}\left(\sum_{i} \sum_{j>i} \mathbb{E}\left[Y_{i}^{2} Y_{j}^{2}\right]+\sum_{i} \sum_{j>i} \sum_{k \neq i, j} \mathbb{E}\left[Y_{i}^{2} Y_{j} Y_{k}\right]\right. \\
& +\sum_{i} \sum_{j>i} \sum_{k \neq i, j} \mathbb{E}\left[Y_{i} Y_{j}^{2} Y_{k}\right] \\
& \left.+\sum_{i} \sum_{j>i} \sum_{k \neq i, j} \sum_{m>k ; m \neq i, j} \mathbb{E}\left[Y_{i} Y_{j} Y_{k} Y_{m}\right]\right) .
\end{aligned}
$$

Denoting by $\left(W_{1}, W_{2}, W_{3}, W_{4}\right)$ a random sample without replacement of four trait values out of $n$ available, we derive

$$
\begin{aligned}
\mathbb{E}\left[D_{n}^{4}\right]= & \frac{\mathbb{E}\left[W_{1}^{4}\right]}{n}-\frac{\mathbb{E}\left[W_{1}^{3} W_{2}\right]}{4 n}+\frac{n^{2}-2 n+3}{n(n-1)} \mathbb{E}\left[W_{1}^{2} W_{2}^{2}\right] \\
& -\frac{2(n-2)(n-3)}{n(n-1)} \mathbb{E}\left[W_{1}^{2} W_{2} W_{3}\right]+\frac{(n-2)(n-3)}{n(n-1)} \mathbb{E}\left[W_{1} W_{2} W_{3} W_{4}\right] .
\end{aligned}
$$

We compute the five fourth-order moments in the last expression using the conditional normality of the random quadruple $\left(W_{1}, W_{2}, W_{3}, W_{4}\right)$ with conditional moments coming from the Ornstein-Uhlenbeck process. Only the covariance is not immediate and is given by

$$
\operatorname{cov}\left[W_{i}, W_{j} \mid y_{n}\right]=\mathbb{E}\left[\mathrm{e}^{-2 \alpha \tau_{i j}^{(n, 4)}} \mid y_{n}\right]-\mathrm{e}^{-2 \alpha U_{n}}, \quad i, j \in\{1,2,3,4\}, i \neq j,
$$

where $\tau_{i j}^{(n, m)}$ is the time to the most recent ancestor for the pair of tips $(i, j)$ among $m$ randomly chosen tips of the Yule $n$-tree. Clearly, all $\tau_{i j}^{(n, 4)}$ have the same distribution as $\tau^{(n)}$, and for

$$
v_{i j}^{(n)}:=\mathbb{E}\left[\mathrm{e}^{-2 \alpha \tau_{i j}^{(n, 4)}} \mid y_{n}\right]
$$

we can find the asymptotics of

$$
\mathbb{E}\left[v_{i j}^{(n)}\right]=\mathbb{E}\left[\mathrm{e}^{-2 \alpha \tau^{(n)}}\right], \quad \mathbb{E}\left[v_{i j}^{(n)} \mathrm{e}^{-2 \alpha U_{n}}\right]=\mathbb{E}\left[\mathrm{e}^{-2 \alpha U_{n}-2 \alpha \tau^{(n)}}\right]
$$

using Lemma 4. Note also that

$$
\mathbb{E}\left[\left(v_{i j}^{(n)}\right)^{2}\right] \sim\left(\mathbb{E}\left[\mathrm{e}^{-2 \alpha \tau^{(n)}}\right]\right)^{2} \quad \text { as } n \rightarrow \infty
$$

This follows from Lemma 11 and Lemma 4 as

$$
\mathbb{E}\left[\left(v_{i j}^{(n)}\right)^{2}\right]=\mathbb{E}\left[\left(\mathbb{E}\left[\mathrm{e}^{-2 \alpha \tau^{(n)}} \mid y_{n}\right]\right)^{2}\right]=\operatorname{var}\left[\mathbb{E}\left[\mathrm{e}^{-2 \alpha \tau^{(n)}} \mid y_{n}\right]\right]+\left(\mathbb{E}\left[\mathrm{e}^{-2 \alpha \tau^{(n)}}\right]\right)^{2} .
$$

Now using the equation for the conditional expectation for $\mathbb{E}\left[Z_{1} Z_{2} Z_{3} Z_{4}\right]$ derived from (6), we obtain the following moment equations:

$$
\begin{gathered}
\mathbb{E}\left[W_{1}^{4} \mid y_{n}\right]=\delta^{4} \mathrm{e}^{-4 \alpha U_{n}}+6 \delta^{2} \mathrm{e}^{-2 \alpha U_{n}}\left(1-\mathrm{e}^{-2 \alpha U_{n}}\right)+3\left(1-\mathrm{e}^{-2 \alpha U_{n}}\right)^{2} \rightarrow 3 \text { as } n \rightarrow \infty, \\
\mathbb{E}\left[W_{1}^{3} W_{2} \mid y_{n}\right]=3 v_{12}^{(n)}-3\left(\delta^{2}-1\right)\left(1-v_{12}^{(n)}\right) \mathrm{e}^{-2 \alpha U_{n}}+\left(\delta^{4}-6 \delta^{2}+3\right) \mathrm{e}^{-4 \alpha U_{n}} \\
\rightarrow 0 \text { as } n \rightarrow \infty,
\end{gathered}
$$




$$
\begin{aligned}
\mathbb{E}\left[W_{1}^{2} W_{2}^{2} \mid y_{n}\right]=1 & +2\left(\delta^{2}-1\right) \mathrm{e}^{-2 \alpha U_{n}}\left(\delta^{4}-6 \delta^{2}+5\right) \mathrm{e}^{-4 \alpha U_{n}}+4\left(\delta^{2}-1\right) v_{12}^{(n)} \mathrm{e}^{-2 \alpha U_{n}} \\
+ & 2\left(v_{12}^{(n)}\right)^{2} \\
\rightarrow & 1 \text { as } n \rightarrow \infty \\
\mathbb{E}\left[W_{1}^{2} W_{2} W_{3} \mid y_{n}\right]= & \left(\delta^{2}-1\right) \mathrm{e}^{-2 \alpha U_{n}}+\left(\delta^{4}-6 \delta^{2}+3\right) \mathrm{e}^{-4 \alpha U_{n}}+2 v_{12}^{(n)} v_{13}^{(n)} \\
& +2\left(\delta^{2}-1\right) \mathrm{e}^{-2 \alpha U_{n}}\left(v_{12}^{(n)}+v_{13}^{(n)}\right)+\left(1+\left(\delta^{2}-1\right) \mathrm{e}^{-2 \alpha U_{n}}\right) v_{23}^{(n)}, \\
\mathbb{E}\left[W_{1} W_{2} W_{3} W_{4} \mid y_{n}\right]= & \left(\delta^{4}-6 \delta^{2}+3\right) \mathrm{e}^{-4 \alpha U_{n}}+v_{12}^{(n)} v_{34}^{(n)}+v_{12}^{(n)} v_{34}^{(n)}+v_{12}^{(n)} v_{34}^{(n)} \\
& +\left(\delta^{2}-1\right) \mathrm{e}^{-2 \alpha U_{n}}\left(v_{12}^{(n)}+v_{13}^{(n)}+v_{14}^{(n)}+v_{23}^{(n)}+v_{24}^{(n)}+v_{34}^{(n)}\right) .
\end{aligned}
$$

Using the Cauchy-Schwarz inequality

$$
0 \leq \mathbb{E}\left[v_{12}^{(n)} v_{13}^{(n)}\right] \leq\left(\mathbb{E}\left[v_{12}^{(n)}\right]\right)^{2}=\left(\mathbb{E}\left[\mathrm{e}^{-2 \alpha \tau^{(n)}}\right]\right)^{2}=o(1),
$$

we obtain $\mathbb{E}\left[W_{1}^{2} W_{2} W_{3}\right] \rightarrow 0$ as $n \rightarrow \infty$. Using Cauchy-Schwarz again for

$$
\mathbb{E}\left[v_{12}^{(n)} v_{34}^{(n)}\right]=\mathbb{E}\left[v_{12}^{(n)} v_{34}^{(n)}\right]=\mathbb{E}\left[v_{12}^{(n)} v_{34}^{(n)}\right],
$$

we find that $\mathbb{E}\left[W_{1} W_{2} W_{3} W_{4}\right] \rightarrow 0$ as $n \rightarrow \infty$. Finally, substituting all of the above into (7), we arrive at $\mathbb{E}\left[D_{n}^{4}\right] \rightarrow 1$.

\section{Appendix A. All moments of $U_{n}$ and $\tau^{(n)}$}

Equation (3) for the Laplace transforms of the random variable $U_{n}$ can be used to calculate the moments of $U_{n}$ using

$$
\mathbb{E}\left[U_{n}^{m}\right]=\left.(-1)^{m}\left(\frac{\partial^{m} \mathbb{E}\left[\mathrm{e}^{-x U_{n}}\right]}{\partial x^{m}}\right)\right|_{x=0} .
$$

For a fixed $n$, we introduce the following notation:

$$
A(x):=\frac{1}{x+1} \cdots \frac{1}{x+n}, \quad b_{m}(x):=\frac{1}{(x+1)^{m}}+\cdots+\frac{1}{(x+n)^{m}},
$$

and $\boldsymbol{b}_{m}(x)=\left(b_{1}(x), \ldots, b_{m}(x)\right)$. Note that $A(0)=1 / n$ ! and $b_{m}(0)=H_{n, m}$ is the $n$th generalized harmonic number of order $m, H_{n, m}=\sum_{i=1}^{n} 1 / i^{m}$. We can write (3) as $\mathbb{E}\left[\mathrm{e}^{-x U_{n}}\right]=$ $n ! A(x)$. Its first derivative with respect to $x$ is $-n ! A(x) b_{1}(x)$, and the second derivative is $n ! A(x)\left(b_{1}(x)^{2}+b_{2}(x)\right)$. For the general recursive formula, we introduce the following notation. We will denote by $\boldsymbol{k}=\left(k_{1}, k_{2}, \ldots\right)$ infinite dimensional vectors with integer-valued components, and write $\boldsymbol{k} \in \mathcal{A}_{m}$ if all $k_{i} \geq 0$ and $|\boldsymbol{k}|:=\sum_{i=1}^{m} k_{i} i=m$. Therefore, $\mathcal{A}_{m}$ represents the set of all possible ways to represent $m$ as a sum of positive integers. We will also use the multi-index notation $\boldsymbol{b}_{m}(x)^{k}=b_{1}(x)^{k_{1}} \cdots b_{m}(x)^{k_{m}}$. Since $A^{\prime}(x)=-A(x) b_{1}(x)$ and $b_{m}^{\prime}(x)=-m b_{m+1}(x)$, we can show by induction that

$$
\frac{\partial^{m}}{\partial x^{m}} \mathbb{E}\left[\mathrm{e}^{-x U_{n}}\right]=(-1)^{m} n ! A(x) \sum_{k \in \mathcal{A}_{m}} c_{\boldsymbol{k}} \boldsymbol{b}_{m}(x)^{\boldsymbol{k}},
$$

where coefficients $c_{\boldsymbol{k}}$ are defined for all vectors $\boldsymbol{k}=\left(k_{1}, k_{2}, \ldots\right)$ with integer-valued components using the recursion

$$
c_{k}=\sum_{j=0}^{m}\left(j k_{j}+1\right) c_{k, j}
$$


with $m=|\boldsymbol{k}|$ and $c_{\boldsymbol{k}, 0}=c_{\left(k_{1}-1, k_{2}, k_{3}, \ldots\right)}, c_{\boldsymbol{k}, j}=c_{\left(k_{1}, \ldots, k_{j}+1, k_{j+1}-1, \ldots\right)}, j \geq 1$. The boundary conditions for the recursion of (9) consist of two parts:

- $c_{k}=0$ if all $k_{i}=0$, or one of the coordinates of the vector $\boldsymbol{k}$ is negative;

- $c_{k}=1$ if $k_{1} \geq 1$ and all other $k_{i}=0$.

From (8), we conclude that

$$
\mathbb{E}\left[U_{n}^{m}\right]=\sum_{k \in \mathcal{A}_{m}} c_{k} \prod_{i=1}^{m} H_{n, i}^{k_{i}} .
$$

The technique for calculating the $m$ th derivative of the Laplace transform of $\tau^{(n)}$ given by (4) is the same but requires new notation $\hat{A}(y):=(y+1) A(y) /(y-1)$ and $\hat{b}_{m}(y):=(y+$ $1)^{m} b_{m} /(y-1)^{m}$. Note that $\hat{A}^{\prime}(y)=-\hat{A}(y) \hat{b}_{1}(y), \hat{b}_{m}^{\prime}(y)=-m \hat{b}_{m+1}(y), \hat{A}(0)=-n !$, and $\hat{b}_{m}(0)=H_{n, m}$ if $m$ is even or $\hat{b}_{m}(0)=H_{n, m}-2$ if $m$ is odd. One can then inductively show that

$$
\frac{\partial^{m}}{\partial y^{m}} \mathbb{E}\left[\mathrm{e}^{-y \tau^{(n)}}\right]=\frac{(-1)^{m} 2 m !}{(n-1)(y-1)^{m-1}}-\frac{(-1)^{m+1}(n+1) !}{n-1} \hat{A}(y)\left(\hat{b}_{1}(y)^{m}+\sum_{\substack{k \in \mathcal{A}_{m} \\ k_{1}<m}} c_{k} \hat{b}_{m}(y)^{k}\right),
$$

with the coefficients $c_{k}$ defined as previously by (9). Therefore, we obtain

$$
\mathbb{E}\left[\tau^{(n)^{m}}\right]=\frac{2 m !}{n-1}-\left(H_{n, 1}-2\right)^{m}+\sum_{\substack{k \in \mathcal{A}_{m} \\ k_{1}<m}} c_{k} \prod_{\substack{i=1 \\ i \text { odd }}}^{m}\left(H_{n, i}-2\right)^{k_{i}} \prod_{\substack{i=1 \\ i \text { even }}}^{m} H_{n, i}^{k_{i}}
$$

Similarly, we can use (5) to write the joint moments of $U_{n}-\tau^{(n)}$ and $\tau^{(n)}$ using

$$
A^{(i, j)}(x):=\frac{1}{x+i+1} \cdots \frac{1}{x+j}, \quad b_{m}^{(i, j)}(x):=\frac{1}{(x+i+1)^{m}}+\cdots+\frac{1}{(x+j)^{m}} .
$$

For $m \geq 1$ and $r \geq 1$, we first obtain

$$
\begin{aligned}
\frac{\partial^{m+r}}{\partial x^{m} \partial y^{r}} & \mathbb{E}\left[\mathrm{e}^{\left.-x\left(U_{n}-\tau^{(n)}\right)-y \tau^{(n)}\right]}\right. \\
= & (-1)^{m+r} \frac{2(n+1) !}{n-1} \\
& \times \sum_{j=1}^{n-1} \frac{A^{(0, j)}(x) A^{(j, n)}(y)}{(j+1)(j+2)}\left(\sum_{k \in \mathcal{A}_{m}} c_{k} b_{m}^{(0, j)}(x)^{k}\right)\left(\sum_{k \in \mathcal{A}_{r}} c_{k} b_{r}^{(j, n)}(y)^{k}\right),
\end{aligned}
$$

and then from the above,

$$
\begin{aligned}
\mathbb{E}\left[\left(U_{n}-\right.\right. & \left.\left.\tau^{(n)}\right)^{m} \tau^{(n)^{r}}\right] \\
= & (-1)^{m+r} \frac{2(n+1)}{n-1} \\
& \times \sum_{j=1}^{n-1} \frac{1}{(j+1)(j+2)}\left(\sum_{k \in \mathcal{A}_{m}} c_{k} \prod_{i=1}^{m} H_{j, i}^{k_{i}}\right)\left(\sum_{k \in \mathcal{A}_{r}} c_{k} \prod_{i=1}^{r}\left(H_{n, i}-H_{j, i}\right)^{k_{i}}\right) .
\end{aligned}
$$




\section{Acknowledgements}

We are grateful to Thomas F. Hansen and Anna Stokowska for helpful suggestions and comments. Special thanks to an anonymous referee for the constructive suggestions to the earlier version of the paper, in particular, for the remark added after Lemma 9.

The research of Serik Sagitov was supported by the Swedish Research Council (grant no. 621-2010-5623). Krzysztof Bartoszek was supported by the Centre for Theoretical Biology at the University of Gothenburg, Svenska Institutets Östersjösamarbete scholarship (grant no. 11142/2013), Stiftelsen för Vetenskaplig Forskning och Utbildning i Matematik (Foundation for Scientific Research and Education in Mathematics), Knut and Alice Wallenbergs travel fund, Paul and Marie Berghaus fund, the Royal Swedish Academy of Sciences, and Wilhelm and Martina Lundgrens research fund.

A previous version of this manuscript formed part of Krzysztof Bartoszek's Doctoral thesis at the Department of Mathematical Sciences, University of Gothenburg, Gothenburg, Sweden.

\section{References}

[1] AdamczaK, R. AND MıŁoś, P. (2014). U -statistics of Ornstein-Uhlenbeck branching particle system. J. Theoret. Prob. 27, 1071-1111.

[2] Adamczak, R. and MiŁoś, P. (2015). CLT for Ornstein-Uhlenbeck branching particle system. Electron. J. Prob. 20, $35 \mathrm{pp}$.

[3] Aldous, D. ANd Popovic, L. (2005). A critical branching process model for biodiversity. Adv. Appl. Prob. 37, 1094-1115.

[4] ANÉ, C. (2008). Analysis of comparative data with hierarchical autocorrelation. Ann. Appl. Statist. 2, $1078-1102$.

[5] ANÉ, C., Ho, L. S. T. AND Roch, S. (2014). Phase transition on the convergence rate of parameter estimation under an Ornstein-Uhlenbeck diffusion on a tree. Preprint. Available at http://arxiv.org/abs/1406.1568.

[6] BartoszeK, K. (2014). Quantifying the effects of anagenetic and cladogenetic evolution. Math. Biosci. 254, 42-57.

[7] BARTOSZEK, K. et al. (2012). A phylogenetic comparative method for studying multivariate adaptation. J. Theoret. Biol. 314, 204-215.

[8] Boettiger, C., Coop, G. AND RalPh, P. (2012). Is your phylogeny informative? Measuring the power of comparative methods. Evolution 66, 2240-2251.

[9] Bohrnstedt, G. W. AND GoldBERGER, A. S. (1969). On the exact covariance of products of random variables. J. Amer. Statist. Assoc. 64, 1439-1442.

[10] Bокма, F. (2010). Time, species, and separating their effects on trait variance in clades. Syst. Biol. 59, 602-607.

[11] Butler, M. A. AND King, A. A. (2004). Phylogenetic comparative analysis: a modeling approach for adaptive evolution. Amer. Naturalist 164, 683-695.

[12] Crawford, F. W. ANd Suchard, M. A. (2013). Diversity, disparity, and evolutionary rate estimation for unresolved Yule trees. Syst. Biol. 62, 439-455.

[13] Edwards, A. W. F. (1970). Estimation of the branch points of a branching diffusion process. J. R. Statist. Soc. $B$ 32, 155-174.

[14] Felsenstein, J. (1985). Phylogenies and the comparative method. Amer. Naturalist 125, 1-15.

[15] GaRland, T., JR. AND IvEs, A. R. (2000). Using the past to predict the present: confidence intervals for regression equations in phylogenetic comparative methods. Amer. Naturalist 155, 346-364.

[16] Garland, T., JR., Midford, P. E. AND Ives, A. R. (1999). An introduction to phylogenetically based statistical methods, with a new method for confidence intervals on ancestral values. Amer. Zool. 39, 374-388.

[17] Gascuel, O. and Steel, M. (2014). Predicting the ancestral character changes in a tree is typically easier than predicting the root state. Syst. Biol. 63, 421-435.

[18] Gernhard, T. (2008). New analytic results for speciation times in neutral models. Bull. Math. Biol. 70, 1082-1097.

[19] Gernhard, T. (2008). The conditioned reconstructed process. J. Theoret. Biol. 253, 769-778.

[20] HANSEN, T. F. (1997). Stabilizing selection and the comparative analysis of adaptation. Evolution 51, 1341-1351.

[21] Hansen, T. F., PienaAR, J. AND OrZack, S. H. (2008). A comparative method for studying adaptation to a randomly evolving environment. Evolution 62, 1965-1977.

[22] Ho, L. S. T. AND ANÉ, C. (2013). Asymptotic theory with hierarchical autocorrelation: Ornstein-Uhlenbeck tree models. Ann. Statist. 41, 957-981. 
[23] Ho, L. S. T. AND ANÉ, C. (2014). A linear-time algorithm for Gaussian and non-Gaussian trait evolution models. Syst. Biol. 63, 397-408.

[24] Ho, L. S. T. AND ANÉ, C. (2014). Intrinsic inference difficulties for trait evolution with Ornstein-Uhlenbeck models. Meth. Ecol. Evol. 5, 1133-1146.

[25] Huelsenbeck, J. P. and Rannala, B. (2003). Detecting correlation between characters in a comparative analysis with uncertain phylogeny. Evolution 57, 1237-1247.

[26] Huelsenbeck, J. P., Rannala, B. and Masly, J. P. (2000). Accommodating phylogenetic uncertainty in evolutionary studies. Science 288, 2349-2350.

[27] Ives, A. R., Midford, P. E. AND GaRland, T., JR. (2007). Within-species variation and measurement error in phylogenetic comparative methods. Syst. Biol. 56, 252-270.

[28] Martins, E. P. ANd Hansen, T. F. (1997). Phylogenies and the comparative method: a general approach to incorporating phylogenetic information into the analysis of interspecific data. Amer. Naturalist 149, 646-667.

[29] Mooers, A. et al. (2012). Branch lengths on birth-death trees and the expected loss of phylogenetic diversity. Syst. Biol. 61, 195-203.

[30] Mossel, E. AND Steel, M. (2014). Majority rule has transition ratio 4 on Yule trees under a 2-state symmetric model. J. Theoret. Biol. 360, 315-318.

[31] Mulder, W. H. AND CraWford, F. W. (2015). On the distribution of interspecies correlation for Markov models of character evolution on Yule trees. J. Theoret. Biol. 364, 275-283.

[32] R Development Core Team (2013). R: A Language and Environment for Statistical Computing. R Foundation for Statistical Computing Vienna, Austria. Available at http://www.r-project.org.

[33] RoHLF, F. J. (2001). Comparative methods for the analysis of continuous variables: geometric interpretations. Evolution 55, 2143-2160.

[34] RohLF, F. J. (2006). A comment on phylogenetic correction. Evolution 60, 1509-1515.

[35] Sagitov, S. AND BartoszeK, K. (2012). Interspecies correlation for neutrally evolving traits. J. Theoret. Biol. 309, 11-19.

[36] Slater, G. J. et al. (2012). Fitting models of continuous trait evolution to incompletely sampled comparative data using approximate Bayesian computation. Evolution 66, 752-762.

[37] STADLER, T. (2008). Lineages-through-time plots of neutral models for speciation. Math. Biosci. 216, 163-171.

[38] STADLER, T. (2009). On incomplete sampling under birth-death models and connections to the sampling-based coalescent. J. Theoret. Biol. 261, 58-68.

[39] Stadler, T. (2011). Simulating trees with a fixed number of extant species. Syst. Biol. 60, 676-684.

[40] STadler, T. and Steel, M. (2012). Distribution of branch lengths and phylogenetic diversity under homogeneous speciation models. J. Theoret. Biol. 297, 33-40.

[41] Steel, M. And McKenzie, A. (2001). Properties of phylogenetic trees generated by Yule-type speciation models. Math. Biosci. 170,91-112.

[42] Stone, E. A. (2011). Why the phylogenetic regression appears robust to tree misspecification. Syst. Biol. 60, 245-260.

[43] Symonds, M. R. E. (2002). The effects of topological inaccuracy in evolutionary trees on the phylogenetic comparative method of independent contrasts. Syst. Biol. 51, 541-553.

[44] Yule, G. U. (1925). A mathematical theory of evolution, based on the conclusions of Dr. J. C. Willis, F. R. S. Philos. Trans. R. Soc. London B 213, 21-87. 